\title{
Diagnosis of Brainstem Abscess in the Cerebritis Stage by Magnetic Resonance Imaging
}

\author{
-Case Report-
}

\author{
Jun-ichi ADACHI, Jiro UKI, Kiyoshi KAZUMOTO, and Fumikazu TAKEDA
}

Neurosurgery Clinic, Saitama Cancer Center, Saitama

\begin{abstract}
A 52-year-old male presented with a brainstem abscess manifesting as high fever, diplopia, and left hemiparesis. Magnetic resonance (MR) imaging with gadolinium diethylenetriamine pentaacetic acid showed the lesion as a ring-like enhanced mass consisting of a necrotic center with surrounding edema, whereas postcontrast computed tomography revealed no such confirmatory findings. He was treated with antibiotics as the lesion had been detected in the acute cerebritis stage. Serial MR images showed that the lesion decreased remarkably in size. MR imaging can detect brain abscess in the earliest inflammatory stage.
\end{abstract}

Key words: brain abscess, brainstem, magnetic resonance imaging, gadolinium diethylenetriamine pentaacetic acid

\section{Introduction}

Neuroimaging methods such as computed tomography (CT) and magnetic resonance (MR) imaging have made the diagnosis of brain abscess easy. ${ }^{1,10,14)}$ However, most previous reports have described the CT and MR imaging findings in the capsule stage. No clinical MR imaging findings of the initial cerebritis stage when the lesion has still not manifested $\mathrm{CT}$ ring-like enhancement have been described.

We describe a case of brainstem abscess detected in the initial cerebritis stage based on MR imaging indications.

\section{Case Report}

A 52-year-old male presented with complaints of high fever, diplopia, and left hemiparesis persisting for 3 days. He had a history of liver cirrhosis but not otolaryngological infection, head trauma, or congenital heart disease.

On admission, he was alert and well oriented, and had neither headache nor nausea. Neurological ex- amination revealed right oculomotor paresis, left hemiparesis including the face, and left hemiataxia. No signs or symptoms of meningeal irritation were evident. Laboratory studies found only mild liver dysfunction, without findings of inflammation such as leukocytosis, elevated erythrocyte sedimentation rate, or increased $\mathrm{C}$-reactive protein. Chest $\mathrm{x}$-ray films disclosed interstitial pneumonia in the bilateral lower pulmonary lobes.

Precontrast CT scans demonstrated an ill-defined low density lesion extending from the right thalamus to the midbrain. No contrast enhancement was shown after intravenous injection of iodine contrast medium $(0.5 \mathrm{mgI} / \mathrm{kg}) \quad$ (Fig. 1). However, $\mathrm{T}_{1^{-}}$ weighted MR images, taken on the same day as the CT scans, revealed a well-demarcated low intensity mass with surrounding local brain edema, and ringlike enhancement following intravenous injection of gadolinium diethylenetriamine pentaacetic acid ( $\mathrm{Gd}$ DTPA) (0.2 mmol $/ \mathrm{kg}$ ) (Fig. 2).

On the 2nd hospital day, severe headaches with nausea began and his consciousness worsened. CT confirmed expansion of the mass and acute obstructive hydrocephalus. Based on the rapid worsening of 

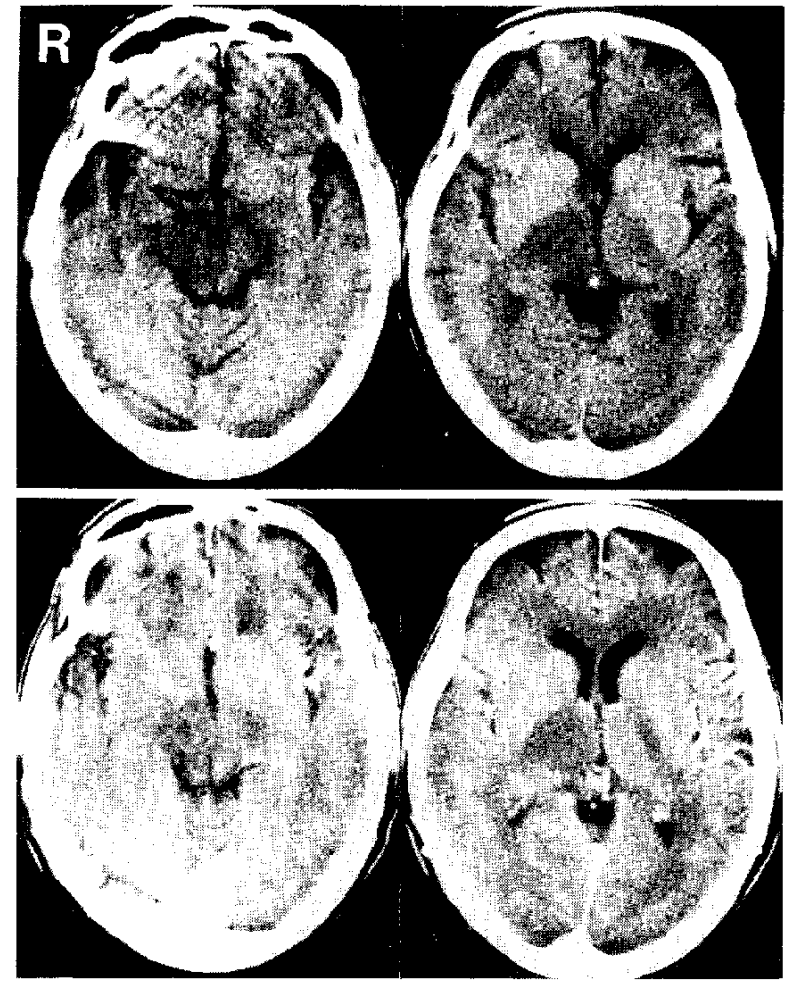

Fig. 1 Precontrast CT scans on admission showing a homogeneous, low density lesion extending from the right thalamus to the midbrain (upper row). Postcontrast CT scans on admission showing no contrast enhancement of the lesion (lower row).

his clinical condition and the image findings which were highly suggestive of brainstem abscess, an emergency ventriculoperitoneal shunt operation was performed to treat his hydrocephalus. The cell count of the ventricular cerebrospinal fluid (CSF) obtained at surgery was $1301 / \mathrm{mm}^{3}$, mainly composed of polynuclear leukocytes. His clinical course associated with fever, the findings of inflammation in the $\mathrm{CSF}$, and the MR imaging ring-like enhancement suggested bacterial meningitis secondary to brainstem abscess.

Bacterial culture of the CSF was negative, so intravenous (i.v.) and intrathecal (i.t.) administration of broad spectrum antibiotics (imipenem/cilastatin $1.0 \mathrm{~g}$ i.v. every $12 \mathrm{hrs}$ and gentamicin $10 \mathrm{mg}$ i.t. every $24 \mathrm{hrs}$ ) was begun immediately after the surgery. His neurological deficits improved rapidly and serial MR images revealed decreased abscess size (Fig. 3). Direct surgical treatment of the brainstem lesion was considered risky, so we selected non-surgical treatment. He continued to receive i.v. and i.t. antibiotic
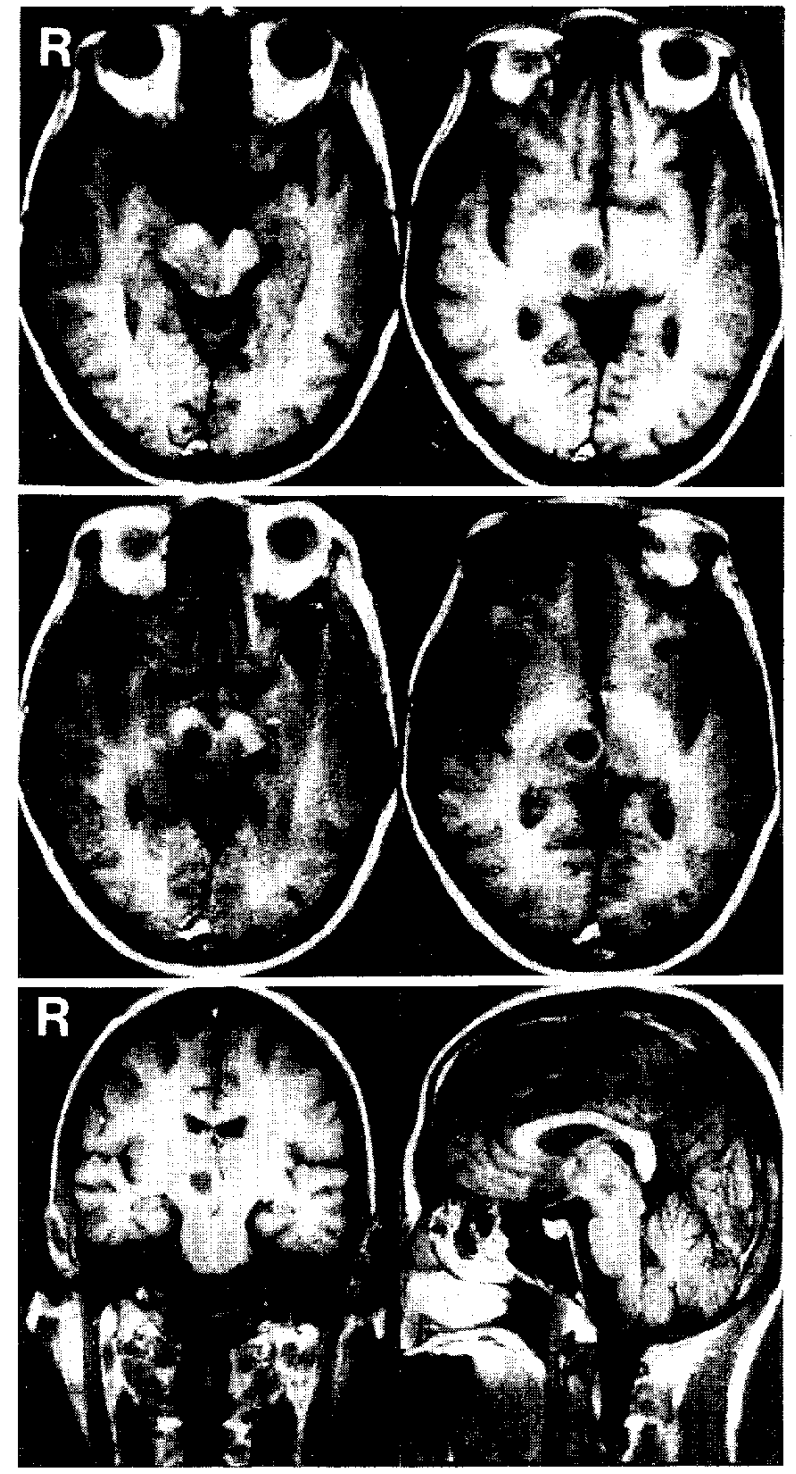

Fig. $2 \mathrm{~T}_{1}$-weighted MR images (spin echo [SE], repetition time $[T R]=500 \mathrm{msec}$, echo time $[\mathrm{TE}]=$ $19 \mathrm{msec}$ ) on admission showing a well-demarcated low intensity mass and swelling of the midbrain (upper row). $\mathrm{T}_{1}$-weighted $\mathrm{MR}$ images with Gd-DTPA (SE, TR $=500 \mathrm{msec}, \mathrm{TE}=$ $19 \mathrm{msec}$ ) on admission showing the central low intensity mass and surrounding edema (middle and lower row). Ring-like enhancement is clearly visible around the low intensity mass. The localization of the lesion is demonstrated threedimensionally.

treatment for about 1 month, followed by oral administration of antibiotics (ofloxacin and chloramphenicol) for about 5 months until CT and MR imaging no longer demonstrated the lesion. Eight months 

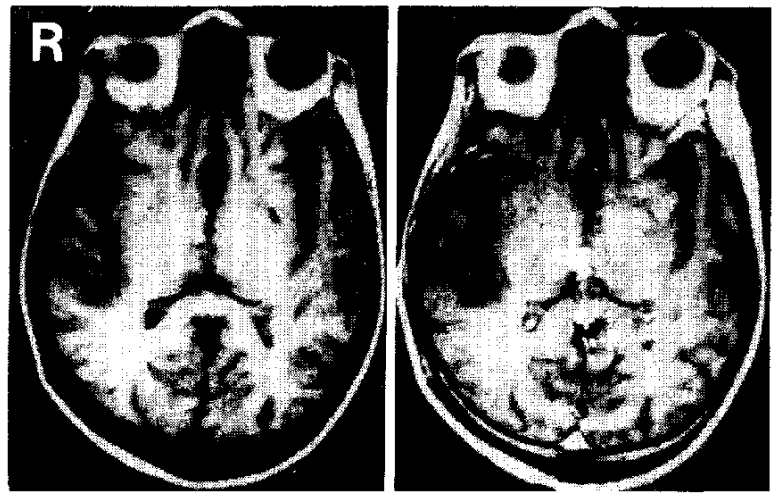

Fig. $3 \mathrm{~T}_{1}$-weighted image $(\mathrm{SE}, \mathrm{TR}=500 \mathrm{msec}, \mathrm{TE}=$ $19 \mathrm{msec}) 4$ months after admission showing the low intensity lesion and the surrounding edema are markedly decreased in size (left), and $T_{1}$-weighted MR image with Gd-DTPA (SE, TR $=500 \mathrm{msec}, \quad \mathrm{TE}=19 \mathrm{msec}$ ) showing the lesion is homogeneously enhanced, compatible with granulation tissue (right).

after diagnosis, he was discharged without neurological deficits except for mild left hemiparesis.

\section{Discussion}

The mortality of patients with brain abscess has decreased to $5 \%$ or less since the development of neuroimaging techniques and modern antibiotic therapy. ${ }^{9)}$ However, the brain abscess remains a critical disease in neurosurgery, since the incidence has not decreased significantly. ${ }^{11)}$ Solitary brainstem abscess was found in about $2 \%$ of all cases with brain abscess. ${ }^{6}$ ) The route of infection is mainly direct extension, usually from middle ear infection, or hematogenous spread from a remote focus. ${ }^{13)}$ Some brain abscesses are of unknown or undetermined origin. We consider that infectious pulmonary lesion had hematogenously metastasized to the brainstem in our case, because the patient had presented with interstitial pneumonia.

CT allows easy diagnosis of brain abscess, accurate determination of the clinical stage, and evaluation of the therapeutic results. ${ }^{2,10)}$ Rosenblum et al. ${ }^{8)}$ reported that the administration of antibiotics during the cerebritis stage of human brain abscess achieved good results. In fact, diagnosis of brain abscess in its earliest stage largely contributes to the effectiveness of treatment, especially the success of antibiotic therapy. However, brain abscess is still difficult to detect at the initial cerebritis stage when ring-like enhancement does not appear on CT scans. ${ }^{7}$ Histological study has shown the early cerebritis stage lasts for about 3 days after bacterial invasion occurs. ${ }^{3)}$ In this period, both the necrotic center composed of the infiltrating inflammatory cells and the surrounding brain edema are formed. ${ }^{1,3)}$ If the abscess is small and capsule formation is not seen, the necrotic center and the surrounding edema may appear as a homogeneous, low density lesion without contrast enhancement on CT scans. ${ }^{3)}$ The initial CT scans of our patient are compatible with the early cerebritis stage.

The high resolution of MR imaging can depict subtle changes in the water content of the associated inflammation, ${ }^{1,10)}$ and can obviously distinguish the different components of a brain abscess as shown in the present case. $T_{1}$-weighted MR imaging showed both the necrotic center and the surrounding edema as low intensity areas, but the former had a lower intensity than the latter. Neovascular hyperplasia and increased vascular permeability occur in the inflammatory zone surrounding the necrotic center, which corresponds to capsule formation. ${ }^{3)} \mathrm{T}_{1}$-weighted MR imaging with Gd-DTPA clearly visualizes this area due to contrast agent accumulation in the region of the defective blood-brain barrier. ${ }^{4,12)} \mathrm{T}_{2}$-weighted MR images demonstrate part of the capsule as a low or iso-intensity rim. ${ }^{5)}$ However, the intensity and thickness of the rim vary with the inflammatory process, ${ }^{5)}$ so that $\mathrm{T}_{1}$-weighted MR images with $\mathrm{Gd}$ DTPA can detect the capsule formation more distinctly.

The definite ring-like enhancement on Gd-DTPAenhanced MR images in the earliest stage of brain abscess formation when no contrast enhancement was observed on $\mathrm{CT}$ scans suggests that MR imaging is a more sensitive detector of this lesion than CT, or that the capsule may develop earlier than previously believed. The correlation between the histology and MR imaging findings at the various stages of brain abscess formation should be investigated further.

\section{References}

1) Brant-Zawadzki $M$, Enzmann $D R$, Placone RC, Sheldon P, Britt RH, Brasch RC, Crooks LA: NMR imaging of experimental brain abscess: Comparison with CT. AJNR 4: 250-253, 1983

2) Britt RH, Enzmann DR: Clinical stage of human brain abscess on serial CT scans after contrast infusion. J Neurosurg 59: 972-989, 1983

3) Britt RH, Enzmann DR, Yeager AS: Neuropathological and computerized tomographic findings in experimental brain abscess. J Neurosurg 55: 590603,1981

4) Grossman RI, Wolf G, Bierry D, McGrath J, Kundel 
H, Aronchick J, Zimmerman RA, Goldberg HI, Bilaniuk LT: Gadolinium enhanced nuclear magnetic resonance images of experimental brain abscess. $J$ Comput Assist Tomogr 8: 204-207, 1984

5) Haimes AB, Zimmerman RD, Morgello S, Weingarten $\mathrm{K}$, Becker RD, Jennis R, Deck MDF: MR imaging of brain abscess. $A J N R$ 10: 279-291, 1989

6) Harvey FH, Carlow TJ: Brainstem abscess and syndrome of acute tegmental encephalitis. Ann Neurol 7: $371-376,1980$

7) Holtas S, Tornquist C, Cronquist S: Diagnostic difficulties in computed tomography of brain abscess. J Comput Assist Tomogr 6: 683-688, 1982

8) Rosenblum ML, Hoff JT, Norman D, Edwards MS, Berg BO: Non operative treatment of brain abscesses in selected high-risk patients. $J$ Neurosurg 52: $217-$ 225,1980

9) Rosenblum ML, Hoff JT, Norman D, Weinstein PR, Pitts LH: Decreased mortality from brain abscesses since the advent of computerized tomography. $J$ Neurosurg 49: 658-668, 1978
10) Salen J, Afshar F: Comparative study of brain abscess by computed tomography and magnetic resonance imaging: Report of two cases. $\mathrm{Br} \mathrm{J}$ Neurosurg 2: 517-522, 1988

11) Samson DS, Clark K: A current review of brain abscess. Am J Med 54: 201-210, 1973

12) Strich G, Hagan PL, Gerber KH, Slutsky RA: Tissue distribution and magnetic resonance spin lattice relaxation effects of gadolinium-DTPA. Radiology 154: 723-726, 1985

13) Weickhardt GD, Davis RL: Solitary abscess of the brainstem. Neurology (Minneap) 14: 918-925, 1964

14) Whelan MA, Hilal SK: Computed tomography as a guide in the diagnosis and follow up of brain abscesses. Radiology 135: 663-671, 1980

Address reprint requests to: J. Adachi, M.D., Biology Division, National Cancer Center Research Institute, 51-1 Tsukiji, Chuo-ku, Tokyo 104, Japan. 